\title{
Radial extracorporeal shock wave therapy is efficient and safe in the treatment of fracture nonunions of superficial bones: a retrospective case series
}

\author{
Paulo Kertzman ${ }^{1}$, Nikolaus B. M. Császár ${ }^{2}$, John P. Furia ${ }^{3}$ and Christoph Schmitz ${ }^{2^{*}}$ (D)
}

\begin{abstract}
Background: A substantial body of evidence supports the use of focused extracorporeal shock wave therapy (fESWT) in the non-invasive treatment of fracture nonunions. On the other hand, virtually no studies exist on the use of radial extracorporeal shock wave therapy (rESWT) for this indication.

Methods: We retrospectively analyzed 22 patients treated with rESWT for fracture nonunions of superficial bones that failed to heal despite initial surgical fixation in most cases. Radial extracorporeal shock wave therapy was applied without anesthesia in three rESWT sessions on average, with one rESWT session per week and 3000 radial extracorporeal shock waves at an energy flux density of $0.18 \mathrm{~mJ} / \mathrm{mm}^{2}$ per session. Treatment success was monitored with radiographs and clinical examinations.

Results: Six months after rESWT radiographic union was confirmed in 16 out of 22 patients (73\%), which is similar to the success rate achieved in comparable studies using fESWT. There were no side effects. The tibia was the most common treatment site (10/22) and 70\% of tibia nonunions healed within 6 months after rESWT. Overall, successfully treated patients showed a mean time interval of $8.8 \pm 0.8$ (mean \pm standard error of the mean) months between initial fracture and commencement of rESWT whereas in unsuccessfully treated patients the mean interval was $26.0 \pm 10.1$ months $(p<0.05)$. In unsuccessful tibia cases, the mean interval was $43.3 \pm 13.9$ months.

Conclusions: Radial extracorporeal shock wave therapy appears to be an effective and safe alternative in the management of fracture nonunions of superficial bones if diagnosed early and no fESWT device is available. The promising preliminary results of the present case series should encourage the implementation of randomized controlled trials for the early use of rESWT in fracture nonunions.
\end{abstract}

Keywords: Bone, Focused extracorporeal shock wave therapy, Fracture, Nonunion, Radial extracorporeal shock wave therapy

\section{Background}

Nonunion refers to the failure of bone fractures to achieve cortical continuity on radiographic studies. The prevalence of nonunion of all fracture types ranges from 2.5 to $46 \%$, and the complication places substantial economic burdens on health systems [1-3]. Surgical

\footnotetext{
* Correspondence: christoph_schmitz@med.uni-muenchen.de

${ }^{2}$ Extracorporeal Shock Wave Research Unit, Chair of Neuroanatomy, Institute of Anatomy, Faculty of Medicine, LMU Munich, Pettenkoferstr 11, D-80336 Munich, Germany

Full list of author information is available at the end of the article
}

fracture stabilization using bone grafts and internal/external fixation has remained the gold standard for treating fracture nonunions. However, these procedures often lead to serious complications including deep infections, persistent wound drainage, hematoma formation, sensory loss, persisting pain, and nonunions $[2,4-6]$.

Hence, there remains a need for efficient therapies that will bring better results more quickly and without major complications. Over the past decades, extracorporeal shock wave therapy (ESWT) has emerged as an efficient, non-invasive and cost-effective alternative to surgery in 
the treatment of fracture nonunions. There are two forms of ESWT available, focused (fESWT) and radial (rESWT) (see [7-9] for detailed reviews). In very brief, fESWT makes use of single acoustic pulses that are generated either with a spark-gap (electrohydraulic principle), a technology similar to a loudspeaker (electromagnetic principle), or piezocrystals (piezoelectric principle). By means of reflectors of certain shape, the acoustic pulses are converted into a focused acoustic pressure wave/shock wave with a point of highest pressure at the desired target within pathological tissue. In case of rESWT, a projectile is fired within a guiding tube that strikes a metal applicator placed on the skin. The projectile generates stress waves in the applicator that transmit pressure waves into tissue. Both focused and radial extracorporeal shock waves are single acoustic impulses with an initial high positive peak pressure between 10 and $100 \mathrm{MPa}$ reached in less than $1 \mu \mathrm{s}$. The positive pressure amplitude is followed by a low tensile amplitude of a few microseconds duration that can generate cavitation. They are further characterized by a short life cycle of approximately 10-20 $\mu$ s and a broad frequency spectrum (see [7-9] for more details).

A total of 40 studies [10-49] have shown an overall success rate of approximately $76 \%$ after 6 months when treating fracture nonunions with fESWT, without major complications (Table 1; a comprehensive overview on details of these studies is provided in Additional file 1). Among these 40 studies were 1 randomized controlled trial (RCT) with a success rate of $71 \%$ after 6 months [10], 2 cohort studies comparing fESWT with surgery, with success rates of 91 [11] and 79\% [12] after 6 months, and 37 case series without control group [13-49]. The RCT [10] and the cohort studies [11, 12] were recently reviewed in detail elsewhere [50].

In contrast, there are only few reports of using rESWT for treating fracture nonunions. We are aware of only one case report study demonstrating the success of rESWT in the consolidation of fracture nonunions involving the base of the second metatarsal in two professional dancers [51].

Acknowledging recent findings (i) from an animal study in vivo demonstrating that radial extracorporeal shock waves (rESWs) can induce new bone formation [52] as well as (ii) from an in vitro study showing that rESWs can induce proliferation of human osteoblast like cells (MG63) [53], the purpose of the present study was to test the following hypothesis: radial extracorporeal shock waves can stimulate bone healing in fracture nonunions of superficial bones.

\section{Methods}

The present study represents a retrospective case series in 22 patients investigating the effects of rESWT on fracture nonunions between 2007 and 2012. The study was approved by the ethics committee of the Medical School of Santa Casa de São Paulo (São Paulo, Brazil) (Reference numbers 12558513.1.0000.5479 CAAE and 232.292 from 27 March 2013). Informed consent was achieved from each patient to participate in this study.

In line with several reports on fESWT for facture nonunions in the literature [11, 37, 39, 43], a nonunion was defined as a fracture that has failed to show continuity of three of four cortices after surgical or nonsurgical treatment for six or more months from the time of the fracture-related injury, or has failed to demonstrate any radiographic change (improvement) for three consecutive months, and is associated with clinical findings consistent with a fracture nonunion (an inability to bear weight on the affected extremity, pain on palpation, or motion at the fracture site for 3 to 6 months or more following the incident traumatic event or the last surgical procedure. Patients included 9 females and 13 males, with a mean age of $35.3 \pm 3.5$ years (mean \pm standard error of the mean) (range 14 to 69) (Table 2). Twenty of these patients had undergone initial surgical treatment of the fracture and presented to the first author of the present study at different time points postoperatively (months to years) due to persisting pain and functional limitations. Fracture nonunion sites in these patients included the clavicle (i.e., collarbone; one patient), ulna (one patient), carpal scaphoid (one patient), tibia (10 patients, one of whom with an infected/atrophic tibia), medial malleolus (two patients), lateral malleolus (one patient), navicular bone of the foot (one patient), second metatarsal bone (one patient), and fifth metatarsal bone (two patients). Surgical procedures included internal plates, nails, and intramedullary/internal screw fixations. The remaining two patients, one with an inferior iliac crest fracture and one with a fibula fracture, presented to the first author of the present study, respectively, 7 and 8 months after the trauma without receiving initial surgery.

All patients received their first rESWT treatment between May 2007 and September 2012. The standard procedure for rESWT in the present study consisted of three consecutive outpatient clinic rESWT sessions in 1-week intervals, although the number of rESWT sessions was adjusted to each patients' individual clinical situation (Table 2). Radial ESWT was performed without anesthesia using a Swiss DolorClast device (Electro Medical Systems, Nyon, Switzerland) and consisted of 3000 radial extracorporeal shock waves per session at the device's highest energy setting (i.e., 4 bar air pressure equivalent to an energy flux density, EFD, of $0.18 \mathrm{~mJ} / \mathrm{mm}^{2}$ ). The "radial" handpiece of the device with its $15-\mathrm{mm}$ applicator was used in all cases. In each case, the point of application was determined prior to rESWT by means of radiographs and 
Table 1 Overview on all studies investigating the effects of focused extracorporeal shock wave therapy (fESWT) for fracture nonunions listed in PubMed (as of March 01, 2017)

\begin{tabular}{|c|c|c|c|c|c|c|c|c|c|c|c|c|c|}
\hline R & Study & $T$ & $n$ & $D$ & $\begin{array}{l}\text { Interval } \\
\text { [months] }\end{array}$ & $\begin{array}{l}\mathrm{SR}_{\mathrm{M} 6} \\
{[\%]}\end{array}$ & $\begin{array}{l}\mathrm{SR}_{\mathrm{T}} \\
{[\%]}\end{array}$ & $N_{S}$ & $\mathrm{Nf}_{E S W} / \mathrm{S}$ & $\begin{array}{l}\mathrm{EFD} \\
{\left[\mathrm{mJ} / \mathrm{mm}^{2}\right]}\end{array}$ & $\begin{array}{l}\mathrm{EFD} \mathrm{T}_{\mathrm{T}} \\
{\left[\mathrm{mJ} / \mathrm{mm}^{2}\right]}\end{array}$ & Relative $\mathrm{EFD}_{\mathrm{T}}$ & KV \\
\hline [10] & Cacchio et al. (2009) & RCT & 84 & EM & 11.1 & 71 & 94 & 4 & 4000 & 0.55 & 8800 & 12.1 & \\
\hline [11] & Furia et al. (2010) & $\mathrm{CH}$ & 23 & $\mathrm{EH}$ & 10.4 & 91 & 91 & 1 & 3000 & 0.35 & 1050 & 1.4 & 26 \\
\hline [12] & Notarnicola et al. (2010) & $\mathrm{CH}$ & 58 & EM & 14.8 & 79 & 79 & 3 & 4000 & 0.09 & 1080 & 1.5 & \\
\hline [13] & Valchanou and Michailov (1991) & CS & 79 & $\mathrm{EH}$ & 20.2 & & 85 & 1 & 2500 & & & & \\
\hline [14] & Schleberger and Senge (1992) & CS & 4 & $\mathrm{EH}$ & $\geq 5$ & 75 & 75 & 1 & 2000 & & & & 18 \\
\hline [15] & Heinrichs et al. (1993) & $\mathrm{CS}$ & 53 & EM & & & 67 & 1 & 5750 & & & & \\
\hline [16] & Diesch \& Haupt (1997) & CS & 172 & EH, EM & & 66 & 66 & 1 & 2500 & 0.33 & 813 & 1.1 & \\
\hline [17] & Haupt (1997) & $\mathrm{CS}$ & 100 & $\mathrm{EH}$ & & & 65 & & & & & & \\
\hline [18] & Haupt (1997) & CS & 87 & $\mathrm{EH}$ & & & 67 & 1 & 2000 & & & & 21 \\
\hline [19] & Vogel et al. (1997) & CS & 52 & & & & 52 & & & & & & \\
\hline [20] & Vogel et al. (1997) & CS & 48 & EM & 12 & & 60 & 1 & 3000 & 0.6 & 1800 & 2.5 & \\
\hline [21] & Beutler et al. (1999) & CS & 25 & $\mathrm{EH}$ & 9 & 41 & 41 & 2 & 2000 & & & & 18 \\
\hline [22] & Rompe et al. (2001) & CS & 43 & EM & 11.4 & & 72 & 1 & 3000 & 0.6 & 1800 & 2.5 & \\
\hline [23] & Schaden et al. (2001) & CS & 115 & $\mathrm{EH}$ & 6 & & 76 & 1 & 6500 & 0.33 & 2113 & 2.9 & 24 \\
\hline [24] & Wang et al. (2001) & CS & 72 & $\mathrm{EH}$ & & 61 & 80 & 1 & 3500 & 0.55 & 1908 & 2.6 & \\
\hline [25] & Küfer et al. (2002) & CS & 4 & EM & $\geq 6$ & & 75 & 3 & 2500 & 0.12 & 900 & 1.2 & \\
\hline [26] & Schatz et al. (2002) & CS & 31 & EM & 10.5 & & 68 & 1 & 6000 & 1.5 & 9000 & 12.3 & \\
\hline [27] & Biedermann et al. (2003) & CS & 73 & $\mathrm{EH}$ & 6 & & 56 & 1 & 2900 & 0.7 & 2030 & 2.8 & \\
\hline [28] & Chooi and Penafort (2004) & CS & 5 & $\mathrm{EH}$ & 26.6 & 40 & 40 & 1 & 4000 & & & & 25 \\
\hline [29] & Schaden et al. (2004) & CS & 613 & $\mathrm{EH}$ & 16.1 & 76 & 76 & 1 & 3000 & 0.38 & 1140 & 1.6 & \\
\hline [30] & Bara and Synder (2007) & CS & 81 & $\mathrm{EH}$ & 8 & 83 & 83 & 1 & 2250 & & & & 20 \\
\hline [31] & Taki et al. (2007) & CS & 5 & $\mathrm{EH}$ & 12 & 100 & 100 & 1 & 3000 & 0.35 & 1050 & 1.4 & 25 \\
\hline [32] & Endres et al. (2008) & CS & 1 & EM & 9 & 100 & 100 & 4 & & 0.4 & & & \\
\hline [33] & Cacchio et al. (2009) & CS & 34 & EM & 6 & 77 & 77 & 4 & 4000 & 0.4 & 6400 & 8.8 & \\
\hline [34] & Moretti et al. (2009) & CS & 204 & EM & & 85 & 85 & 1 & 4000 & 0.66 & 2640 & 3.6 & \\
\hline [35] & Wang et al. (2009) & CS & 42 & $\mathrm{EH}$ & 15 & 79 & 79 & 1 & 6000 & 0.62 & 3720 & 5.1 & 28 \\
\hline [36] & Xu et al. (2009) & CS & 69 & EM & 12.5 & 65 & 76 & 1 & 6500 & 0.59 & 3835 & 5.3 & 26 \\
\hline [37] & Elster et al. (2010) & CS & 192 & $\mathrm{EH}$ & 16.8 & 72 & 72 & 1 & 7000 & 0.39 & 3100 & 4.2 & 27 \\
\hline [38] & Alvarez et al. (2011) & CS & 32 & $\mathrm{EH}$ & 7 & 73 & 95 & 1 & 2000 & 0.37 & 730 & 1 & \\
\hline [39] & Stojadinovic et al. (2011) & CS & 349 & $\mathrm{EH}$ & & 81 & 81 & 1 & 7000 & 0.5 & 3500 & 4.8 & 27 \\
\hline [40] & Vulpiani et al. (2012) & CS & 143 & EM & 14.1 & & 56 & 6 & 2750 & 0.55 & 8301 & 11.2 & \\
\hline [41] & Czarnowska-Cubała et al. (2013) & CS & 31 & $\mathrm{EH}$ & 22.6 & 39 & 39 & 1 & 3000 & & & & 20.5 \\
\hline [42] & Alkhawashki (2015) & CS & 44 & $\mathrm{EH}$ & 11.9 & & 76 & 1 & 3000 & & & & 26 \\
\hline [43] & Kuo et al. (2015) & CS & 22 & $\mathrm{EH}$ & 10.5 & & 64 & 1 & 6000 & 0.58 & 3480 & 4.8 & 28 \\
\hline [44] & Haffner et al. (2016) & CS & 52 & $\mathrm{EH}$ & 15.6 & 89 & & 1 & 4000 & 0.4 & 1600 & 2.2 & \\
\hline [45] & Ikeda et al. (1999) & CS & 6 & a & 14 & & 67 & & & & & & \\
\hline [46] & Ikeda (2009) & $\mathrm{CS}$ & 8 & a & & & 63 & & & & & & \\
\hline
\end{tabular}

$R$ reference number, $T$ type of study, $R C T$ randomized controlled trial, $C H$ cohort study, $C S$ case series, $n$ number of patients treated with fESWT, $D$ type of fESWT device, $E H$ electrohydraulic fESWT device, EM electromagnetic fESWT device, interval interval between initial fracture and first nonunion treatment (fESWT or other), $S R_{M \sigma}$ success rate after 6 months, $S R_{T}$ total success rate, $N_{S}$ number of fESWT sessions, $N_{f E S W / S}$ number of focused extracorporeal shock waves per session, $E F D$ energy flux density of the applied fESWs, EFD total energy flux density, relative $E F D_{T}$ multiple of EFD compared to the EFD $_{T}$ applied in [38], KV kilovolt. Note that for $N_{S}, N_{\mathrm{fESW}} / \mathrm{S}, \mathrm{EFD}, \mathrm{EFD}$, and KV average values are provided in case more than one fESWT protocol was used in the corresponding study (details are provided in Additional file 1). In case no data are shown, they were either not provided in the corresponding study or could not be calculated (details are provided in Additional file 1). Note that the following studies are not listed in the table: [47] (same data as in [19]), [48] (same data as in [22]) and [49] (dataset included in [30])

${ }^{a}$ Extracorporeal shock waves generated by means of explosions 
Table 2 Clinical data and treatment outcome of patients with fracture nonunions enrolled in the present study

\begin{tabular}{|c|c|c|c|c|c|c|c|}
\hline Case \# & Sex & Age (years) & Nonunion site & Fixation & 1 & S & O \\
\hline 1 & M & 38 & Ulna & Internal plate & 7 & 4 & + \\
\hline 2 & M & 30 & Carpal scaphoid & Screw fixation & 5 & 3 & + \\
\hline 3 & $\mathrm{~F}$ & 14 & Tibia & $\begin{array}{l}\text { Intramedullary } \\
\text { nail (locked) }\end{array}$ & 12 & 4 & + \\
\hline 4 & $\mathrm{~F}$ & 21 & Tibia & $\begin{array}{l}\text { Intramedullary } \\
\text { nail (locked) }\end{array}$ & 9 & 5 & + \\
\hline 5 & $\mathrm{~F}$ & 28 & Tibia & $\begin{array}{l}\text { Intramedullary } \\
\text { nail (locked) }\end{array}$ & 7 & 3 & + \\
\hline 6 & $\mathrm{~F}$ & 43 & Tibia and fibula & $\begin{array}{l}\text { Intramedullary } \\
\text { nail (tibia, locked) }\end{array}$ & 5 & 4 & + \\
\hline 7 & M & 46 & Tibia & $\begin{array}{l}\text { Intramedullary } \\
\text { nail (locked) }\end{array}$ & 10 & 2 & + \\
\hline 8 & M & 48 & Tibia & $\begin{array}{l}\text { Intramedullary } \\
\text { nail (locked) }\end{array}$ & 9 & 3 & + \\
\hline 9 & M & 59 & Tibia & $\begin{array}{l}\text { Intramedullary } \\
\text { nail (locked) }\end{array}$ & 10 & 4 & + \\
\hline 10 & $\mathrm{~F}$ & 58 & Medial malleolus & Screw fixation & 14 & 3 & + \\
\hline 11 & M & 69 & Medial malleolus & Screw fixation & 6 & 3 & + \\
\hline 12 & $\mathrm{~F}$ & 15 & Second metarsal & Screw fixation & 6 & 4 & + \\
\hline 13 & M & 19 & Fifth metatarsal & Screw fixation & 17 & 3 & + \\
\hline 14 & M & 20 & Fifth metatarsal & Screw fixation & 9 & 3 & + \\
\hline 15 & M & 15 & Iliac crest & n.a. & 7 & 3 & + \\
\hline 16 & M & 21 & Fibula & n.a. & 8 & 4 & + \\
\hline 17 & $\mathrm{~F}$ & 53 & Clavicle & Internal plate & 4 & 2 & - \\
\hline 18 & $\mathrm{~F}$ & 19 & Tibia & $\begin{array}{l}\text { Intramedullary } \\
\text { nail (locked) }\end{array}$ & 18 & 5 & - \\
\hline 19 & M & 33 & Tibia (infected) & $\begin{array}{l}\text { Intramedullary } \\
\text { nail (locked), } \\
\text { internal plate }\end{array}$ & 66 & 6 & - \\
\hline 20 & M & 37 & Tibia & $\begin{array}{l}\text { Intramedullary } \\
\text { nail (locked) }\end{array}$ & 46 & 5 & - \\
\hline 21 & $\mathrm{~F}$ & 49 & Lateral malleolus & Srew fixation & 6 & 3 & - \\
\hline 22 & M & 41 & $\begin{array}{l}\text { Navicular bone } \\
\text { (foot) }\end{array}$ & Screw fixation & 16 & 3 & - \\
\hline
\end{tabular}

I interval between fracture and first session of radial extracorporeal shock wave therapy (rESWT), $S$ number of rESWT sessions, $O$ outcome, + positive clinical outcome, - negative clinical outcome

palpation. After rESWT, patients with metatarsal fractures were advised to wear an open toe cast shoe for 3 weeks whereas patients with tibia fractures were advised to refrain from weight bearing and any sports activities for 4 weeks. During the following 6 months patients were monitored by means of radiographs and clinical examinations.

A positive therapy outcome was defined by radiographic consolidation and absence of both pain and functional limitations during normal weight loading 6 months after commencement of rESWT. Adverse events during and after rESWT were documented.

Mean and standard error of the mean (SEM) of patient's age, number of rESWT sessions, and interval between the initial fracture and the first rESWT session were separately calculated for patients with positive clinical outcome (Group rESWT+) and patients with negative clinical outcome (group rESWT-). The ShapiroWilk normality test was used to determine whether the distribution of patients' age, the interval between the initial fracture and the first rESWT session, and the number of rESWT sessions of the patients in Groups rESWT+ and rESWT- were consistent with a Gaussian distribution. Differences between groups were tested with (i) Fisher's exact test for the relative numbers of female and male patients, (ii) Student's $t$ test for the mean age of the patients and the mean interval between the initial fracture and the first rESWT session, and (iii) non-parametric Mann Whitney test for the mean number of rESWT sessions. In all analyses, an effect was considered statistically significant if its associated $p$ value was smaller than 0.05 . All calculations were performed using GraphPad Prism (version 5.00 for Windows, GraphPad Software, San Diego, CA, USA).

\section{Results}

Six months after receiving rESWT, 16 out of 22 patients $(16 / 22=73 \%)$ had a positive outcome defined by radiographic bone consolidation and the absence of both pain and functional limitations during normal weight loading. Treatments were well tolerated by all patients, with bearable pain during treatment devoid of anesthesia being the main complication reported. Neither skin injuries nor hematomas nor ecchymosis were observed during or after any of the rESWT sessions.

There were no statistically significant age or gender differences observed among patients with positive outcome (average age: $34.0 \pm 4.5$ years; mean \pm SEM; $n=16$; 6 females, 10 males) or negative outcome (average age: $38.7 \pm 5.0$ years; $n=6$; three females and three males) (age: $p=0.565$; gender: $p=0.655$ ) (Fig. 1a). Nor was there a statistically significant difference in the mean number of rESWT sessions applied between the groups with positive (3.4 \pm 0.2 sessions) and negative outcome $(4.0 \pm 0.6$ sessions) $(p=0.253)$ (Fig. 1b). On the other hand, there was a statistically significant difference between Groups rESWT+ and rESWT - with respect to the time interval between initial trauma and first application of rESWT: in the group with positive clinical outcome this time interval was $8.8 \pm 0.8$ months whereas in the group with negative clinical outcome on average $26.0 \pm 10.1$ months had passed between bone fracture and rESWT commencement ( $p=0.010)$ (Fig. 1c).

In the present study, the tibia was the most frequent fracture nonunion site, and in 7 out of 10 tibia cases (7/ $10=70 \%)$ rESWT yielded positive results. An impressive case is shown in Fig. 2. This was a 14-year-old girl who suffered in a traffic accident a combined fracture of the left tibia and fibula (Fig. 2a). Initial surgery was 


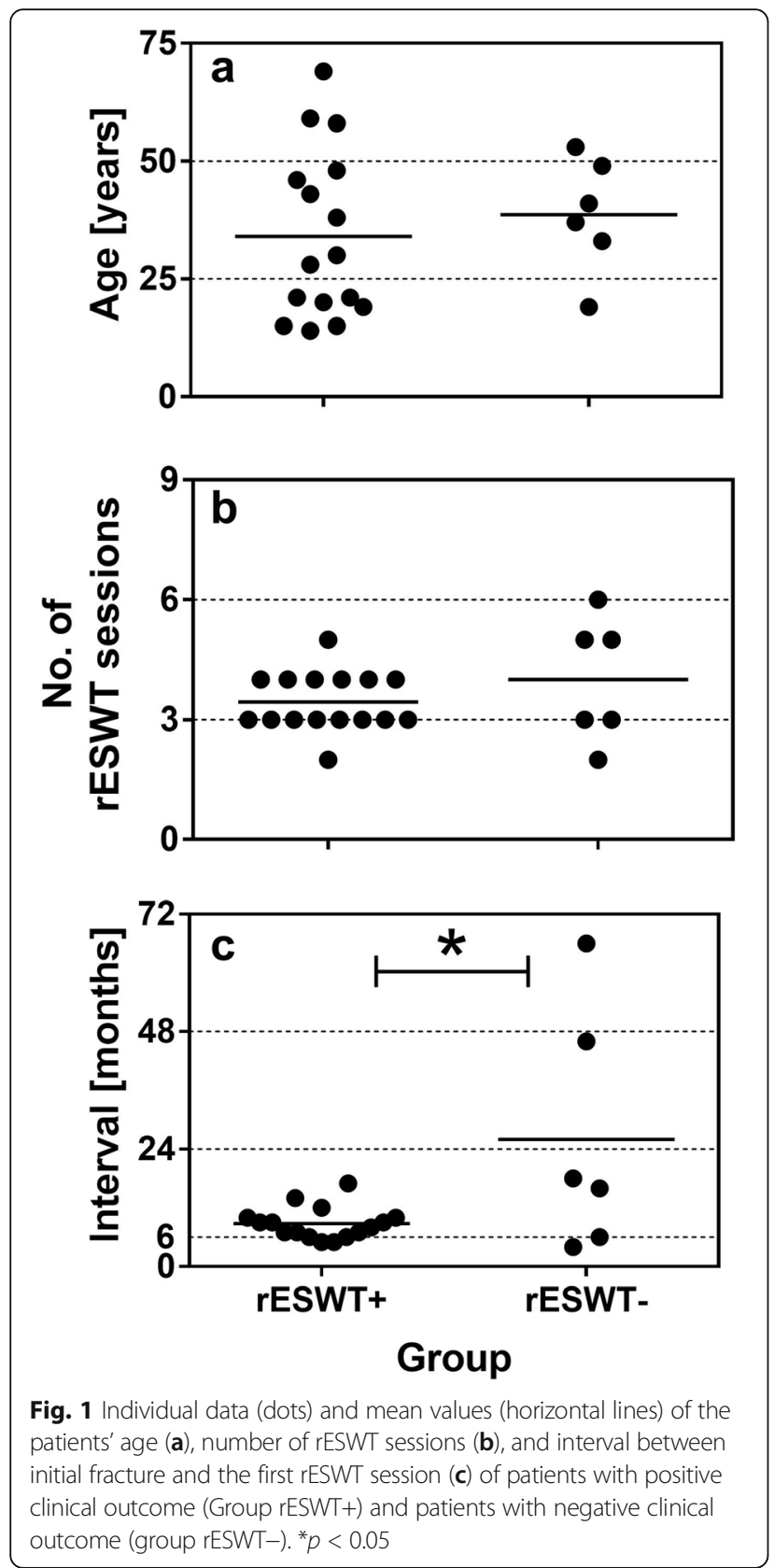

performed using locked intramedullary nailing of the tibia (Fig. 2b). Twelve months after the initial fracture consolidation was still not achieved, and the patient experienced severe pain during walking (Fig. 2c, d). At this time point, a series of four rESWT sessions was started. Six months later, the patient was pain-free during walking, and radiographic consolidation was achieved (Fig. 2e). Another 12 months later the intramedullary nail was removed (Fig. 2f).

Regarding those tibia patients unresponsive to rESWT, it is noteworthy that their time interval between fracture and rESWT commencement was on average approximately

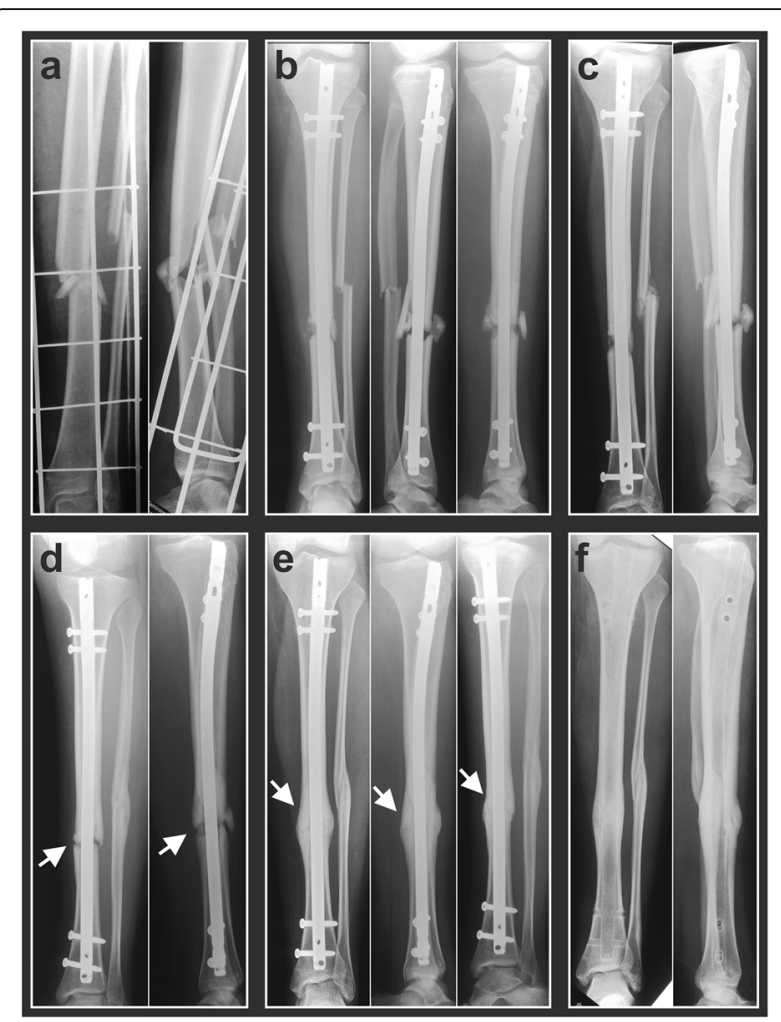

Fig. 2 Radiographs of a 14-year-old-girl who suffered in a traffic accident a combined fracture of the left tibia and fibula, showing the situation immediately after the accident (a) as well as 2 weeks (b), 3 months (c), and 12 months (d) after the accident. Fracture consolidation was still not achieved (arrows in $\mathbf{d}$ ), and the patient experienced severe pain during walking. At that time, a series of four rESWT sessions was started. Six months later (e), the patient was pain-free during walking, and radiographic consolidation was achieved (arrows in e). Another 12 months later, the intramedullary nail was removed ( $f$ )

five times higher compared to the group with positive clinical outcome (i.e., $43.3 \pm 13.9$ months vs. $8.9 \pm 0.9$ months, respectively). Furthermore, one of the unsuccessfully treated patients showed a tibia atrophy together with a medical history comprising eight failed previous surgeries during an interval of 66 months, the longest interval among all patients in the present study.

The remaining nine cases with positive clinical outcome involved the ulna (one patient), carpal scaphoid (one patient), inferior iliac crest (one patient), fibula (one patient), medial malleolus (two patients), second metatarsal bone (one patient), and fifth metatarsal bone (two patients). In contrast, in one of each case involving the clavicle, lateral malleolus and navicular bone of the foot rESWT failed to achieve bony union for unknown reasons.

\section{Discussion}

The present retrospective study, to our knowledge, represents the first pilot case series involving more than 
two patients with fracture nonunions treated with rESWT. Sixteen out of 22 patients (73\%) were successfully treated with rESWT, as confirmed by radiographic consolidation at the 6-month follow-up. There were no complications. Anesthesia during treatment was not necessary. Nonunions of the tibia were the most common and $70 \%$ of the tibia nonunions healed within 6 months after rESWT.

Both focused and radial extracorporeal shock waves are single acoustic impulses with an initial high positive peak pressure between 10 and $100 \mathrm{MPa}$ reached in less than $1 \mu \mathrm{s}$ (reviewed in [9]). The positive pressure amplitude is followed by a low tensile amplitude of a few microseconds duration that can generate cavitation [54-56]. They are further characterized by a short life cycle of approximately 10-20 $\mu$ s and a broad frequency spectrum [57]. Focused ESWs differ from rESWs in the penetration depth into the tissue, some physical characteristics, and the technique for generating them [7, 9, 57].

We noted an association between prolonged time interval between fracture and rESWT procedure and failure of treatment. This discrepancy among groups with positive and negative clinical outcome was most pronounced in tibia cases, where unsuccessfully treated patients displayed on average approximately five times longer intervals than successfully treated patients. In this respect, it is important to point out that the interval (together with the specific type of bone affected) has been identified as the most predictive prognostic indicator for fracture nonunion healing at 6 months following fESWT [39]; i.e., with intervals longer than 11 months significantly reducing the likelihood of a positive clinical outcome (see also [44]). In the present study, the mean interval among the few rESWT resistant patients and particularly among therapy resistant tibia patients exceeded this 11-month threshold, respectively, two- and fourfold. The interval is therefore of relevance when treating fracture nonunions with ESWT, both in clinical routine and in future RCTs on this topic.

Some reports in the literature [44, 58-60] referred to the following definition of fracture nonunion provided by the United States Food and Drug Administration (FDA) [61]: "A non-union study would include patients whose fractures have not healed for a minimum of nine months and who have not undergone surgical intervention during the previous three months." It is important to note that only two out of the 37 studies (5.4\%) on fESWT for fracture nonunions listed in Table 1 [22, 44] adhered to this definition. In contrast, in 22 of these studies (59.5\%) a minimum time interval of 6 months was applied to define fracture nonunions (as was done in the present study and in the only prior report involving the use of rESWT for treating fracture nonunions [51] discussed below). Furthermore, in 9 out of the 37 studies
(24.3\%) listed in Table 1, no definition of fracture nonunions was provided, and in four of these studies different definitions of fracture nonunions were applied (details are outlined in Additional file 1). Several reasons for this discrepancy are conceivable. Specifically, the definition by FDA was provided without any reference to the academic literature and was provided in a draft of a Guidance Document that was released for comment in 1998 but has never become an official FDA Guidance Document. Accordingly, the definition provided above may have never become official opinion by FDA.

To our knowledge, there exists only one prior report involving the use of rESWT for treating fracture nonunions [51]. In this small case series of two professional dancers, rESWT was successfully used to achieve fracture union of the base of the second metatarsal bone. One fracture nonunion healed 3 months post treatment and the other healed 6 months post treatment. In the present study, the same treatment protocol as used by Silk et al. [51] was applied (i.e., three rESWT sessions at 1-week intervals; 3000 radial extracorporeal shock waves per session), although with a slightly lower energy flux density (i.e., 0.18 vs. $0.20 \mathrm{~mJ} / \mathrm{mm}^{2}$ ), and positive clinical outcome was achieved in the majority of cases. Therefore, rESWT appears to be an effective and safe treatment option for fracture nonunions of superficial bones in case a fESWT device is not available.

With exception of one study [51], all clinical studies on the effects of ESWT in the treatment of fracture nonunions have so far been conducted using fESWT (Table 1) [10-49]. This evidence listed in PubMed as of 01 March 2017 includes one prospective RCT (level of evidence, 1) comparing two different fESWT protocols with surgery [10], 2 cohort studies also comparing fESWT with surgery [11, 12], 34 case series without control group [13-46], and 3 double publications of data [47-49]. Collectively, these 40 studies have shown a mean success rate of approximately $76 \%$ after 6 months. However, this mean success rate must be interpreted cautiously because of substantial heterogeneity of these studies with respect to the treatment site and the fESWT protocol used (note that in some studies [17, 18, 25, 34], the treatment site was not specified). The success rate reported in the RCT comparing fESWT with surgery [10] cannot directly be compared to the results of the present study because in the RCT, $53 \%$ of the treatment sites (67/ 126) were the tibia ( $48 \%$ in the present study) but $27 \%$ $(34 / 126)$ were the femur, $12 \%(15 / 126)$ were the ulna, and $8 \%(10 / 126)$ were the radius (femur and radius were not addressed in the present study). The same applies to the success rates reported in the cohort studies comparing fESWT with surgery because in these studies, only patients suffering from fracture nonunions of, respectively, the proximal fifth metatarsal [11] (only two patients in the 
present study) or the carpal scaphoid [12] (only one patient in the present study) were included. On the other hand, Elster et al. [37] reported for 192 fracture nonunions of the tibia a success rate of $72 \%$ at 6 months after fESWT, which is close to the success rate reported in the present study. Very recently, Haffner et al. [44] reported for 52 fracture nonuions of the tibia a success rate of $89 \%$ at 6 months after fESWT, which is higher than the success rate reported in the present study.

Based on the evidence outlined above, it appears reasonable to propose a "therapy of first choice" recommendation for the treatment of fracture nonunions using ESWT (Fig. 3). Beforehand, it must be stressed that ESWT for nonunions, regardless of whether fESWT or rESWT, must be considered a medical treatment to be performed by medical doctors with exact knowledge about orthopedic diagnostic of nonunion and capacity to decide the best treatment option and possible complications. This is because pain relief must not be used as sole clinical endpoint in these cases; mainly but not exclusively due to the analgesic effects of ESWT, usually causing patients to mobilize the affected limb prior to achieving bony consolidation. The recommendation outlined in Fig. 3 considers both superficial and deep as well as early and late fracture nonunions. In either of these scenarios, as soon as possible after correct fracture nonunion diagnosis, early fESWT if available should be chosen over rESWT or surgery. If fESWT is not available and the bone with the fracture nonunion is superficial, early rESWT instead of surgical procedures should be opted for. If fESWT or rESWT is not successful in treating fracture nonunions of superficial bones then surgical procedures should be performed.

This recommendation seems to be justified not only from the preliminary evidence presented in the present study but also from the increasing evidence stemming from concerted basic research efforts into the mechanisms of rESWT over the last years: (i) the application of rESWT in deep indications such as nonunions of the femur or avascular hip necrosis is contraindicated simply because rESWs cannot reach the intended tissues [55]. On the other hand, radial extracorporeal shock waves can reach superficial bones (including those listed in Table 2), and the results of the present study suggest that rESWT is a safe and effective treatment for fracture nonunions of superficial bones. (ii) For both rESWs and fESWs, new bone formation was demonstrated in animal models in vivo [52, 62]. (iii) For both rESWs and fESWs, induction of proliferation of human osteoblast like cells or primary human osteoblasts was shown in vitro [53, 63].

The present study is an audit of retrospectively collected data and has therefore inherent limitations. First, there was no randomization and no surgery arm to

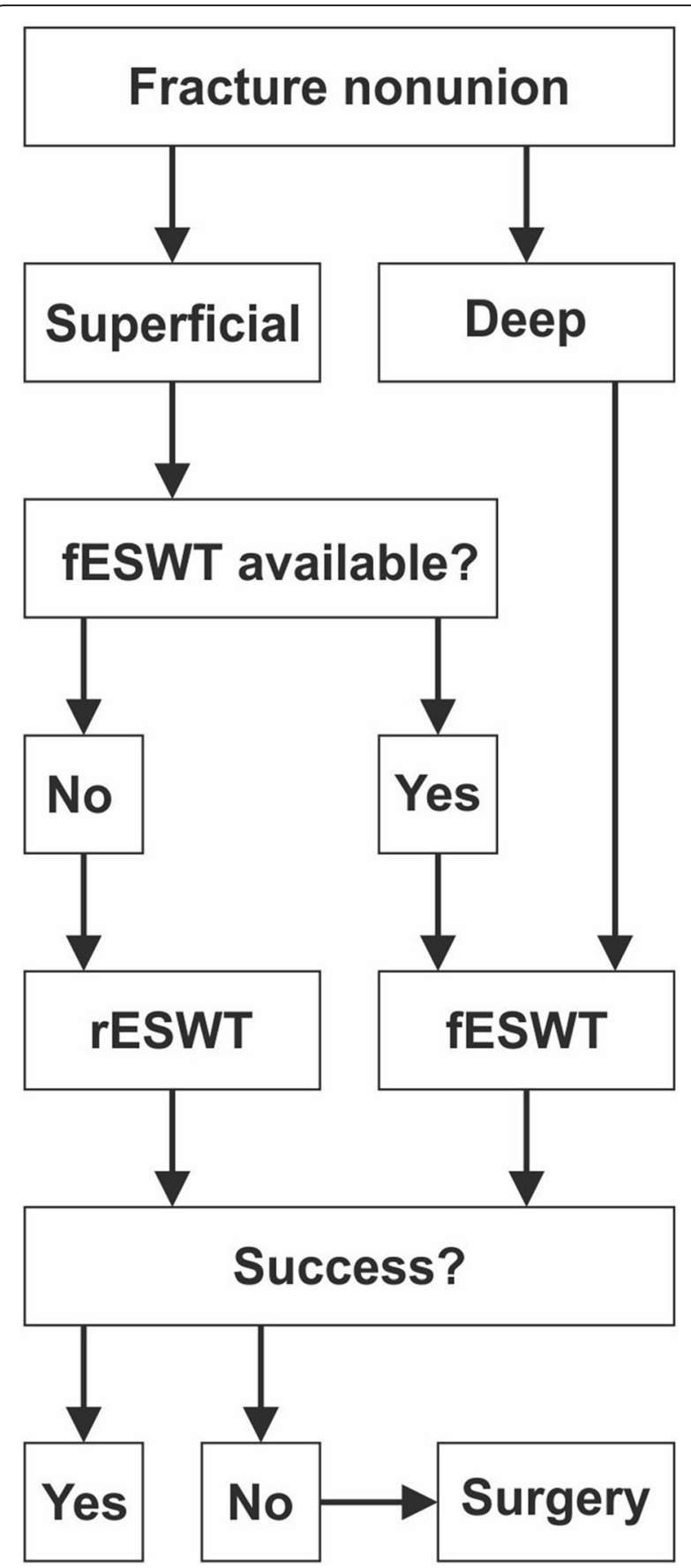

Fig. 3 Proposed decision tree for the treatment of fracture nonunions with extracorporeal shock waves based on the evidence published so far. fESWT focused extracorporeal shock wave therapy, rESWT radial extracorporeal shock wave therapy

this study; however, the same was the case in $92 \%$ of all studies on fESWT for fracture nonunions (34 out of 37 studies without double publication of data). Second, the small number of patients could potentially confound the clinical results. Third, treatment success was determined at 6 months after rESWT, i.e., no total success rate of 
rESWT (regardless of the follow-up interval) for fracture nonunions could be determined. Fourth, no test of other imaging modalities of fracture nonunions such as computer tomography or scintigraphy was performed; however, the symptoms and imaging findings used to define positive clinical outcome in the present study are generally accepted and considered appropriate for this condition.

\section{Conclusions}

The results of the present retrospective study suggest that rESWT is a safe and effective treatment for fracture nonunions of superficial bones. For this reason, medical doctors should consider rESWT prior to surgical intervention in the management of fracture nonunions of superficial bones in case fESWT is not available. In any case, ESWT should be performed as soon as possible after correct diagnosis of fracture nonunion. The promising results of the present retrospective study should encourage the implementation of RCTs using early rESWT in the treatment of fracture nonunions of superficial bones.

\section{Additional file}

Additional file 1: Details of all studies on focused extracorporeal shock wave therapy (fESWT) for fracture nonunions listed in PubMed (as of March 01, 2017). (DOCX $76 \mathrm{~kb})$

\begin{abstract}
Abbreviations
CH: Cohort study; CS: Case series; D: Type of fESWT device; EFD: Energy flux density; EFDT: Total energy flux density; EH: Electrohydraulic fESWT device; EM: Electromagnetic fESWT device; ESWT: Extracorporeal shock wave therapy; FDA: United States Food and Drug Administration; fESWs: Focused extracorporeal shock waves; fESWT: Focused extracorporeal shock wave therapy; I: Interval between fracture and first session of radial extracorporeal shock wave therapy; KV: Kilovolt; $\mathrm{mJ} / \mathrm{mm}^{2}$ : millijoule per squared millimeter; MPa: Megapascal; n: Number of patients treated with fESWT; n.a.: Not applicable; $\mathrm{N}_{\mathrm{fESW}} / \mathrm{s}$ : Number of focused extracorporeal shock waves per session; $\mathrm{N}_{\mathrm{S}}$ : Number of fESWT sessions; O: Outcome; R: Reference number; RCT: Randomized controlled trial; rESWs: Radial extracorporeal shock waves; rESWT-: Patients with negative clinical outcome after rESWT; rESWT: Radial extracorporeal shock wave therapy; rESWT+: Patients with positive clinical outcome after rESWT; S: Number of sessions; SEM: Standard error of the

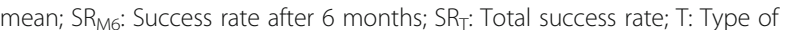
study; $\mu \mathrm{s}$ : Microsecond
\end{abstract}

\section{Acknowledgements}

We thank Dr. Hiroshi Kimura for translating reference no. 45 that was published in Japanese language.

\section{Funding}

This study has not received any financial support or funding.

\section{Availability of data and materials}

The datasets used and analyzed during the current study are available from the corresponding author on reasonable request, taking into account any confidentiality.

\section{Authors' contributions}

PK carried out the conception and design of the study and acquisition and interpretation of data. CS and NBMC participated in data analysis, carried out the interpretation of data, and drafted the manuscript. JPF revised the manuscript critically for important intellectual content. All authors have read and approved the final manuscript.

\section{Ethics approval and consent to participate}

This study was approved by the ethics committee of the Medical School of Santa Casa de São Paulo (São Paulo, Brazil) (Reference numbers 12,558,513.1.0000.5479 CAAE and 232.292 from 27 March 2013). Informed consent was achieved from each patient to participate in this study.

\section{Consent for publication}

The patient whose X-ray images are shown in Fig. 2 has explicitly granted permission to publish these images.

\section{Competing interests}

PK and NBMC declare that no competing interests exist. JPF is the Deputy Editor for the Journal of Orthopedic Surgery and Research. CS serves as a paid consultant for and receives benefits from Electro Medical Systems (Nyon, Switzerland), the manufacturer and distributor of the Swiss DolorClast radial shock wave device. However, Electro Medical Systems had no role in study design, data collection and analysis, decision to publish, or preparation of this manuscript. No other potential conflicts of interest relevant to this article were reported.

\section{Publisher's Note}

Springer Nature remains neutral with regard to jurisdictional claims in published maps and institutional affiliations.

\section{Author details}

${ }^{1}$ Departamento de Ortopedia, Santa Casa de São Paulo, São Paulo, SP, Brazil. ${ }^{2}$ Extracorporeal Shock Wave Research Unit, Chair of Neuroanatomy, Institute of Anatomy, Faculty of Medicine, LMU Munich, Pettenkoferstr 11, D-80336 Munich, Germany. ${ }^{3}$ SUN Orthopaedics and Sports Medicine, Division of Evangelical Community Hospital, 900 Buffalo Road, Lewisburg, PA 17837, USA.

Received: 23 April 2017 Accepted: 20 October 2017

Published online: 06 November 2017

\section{References}

1. Sen MK, Miclau T. Autologous iliac crest bone graft: should it still be the gold standard for treating nonunions? Injury. 2007;38:S75-80.

2. Hak DJ, Fitzpatrick D, Bishop JA, Marsh JL, Tilp S, Schnettler R, et al. Delayed union and nonunions: epidemiology, clinical issues, and financial aspects. Injury. 2014:45:S3-7.

3. Kostenuik P, Mirza FM. Fracture healing physiology and the quest for therapies for delayed healing and nonunion. J Orthop Res. 2017:35:213-23.

4. Younger EM, Chapman MW. Morbidity at bone graft donor sites. J Orthop Trauma. 1989:3:192-5.

5. Warren SB, Brooker AF Jr. Intramedullary nailing of tibial nonunions. Clin Orthop Relat Res. 1992:285:236-43.

6. Wang CJ, Chen HS, Chen CE, Yang KD. Treatment of nonunions of long bone fractures with shock waves. Clin Orthop Relat Res. 2001;387:95-101.

7. Gerdesmeyer L, Maier M, Haake M, Schmitz C. Physikalisch-technische Grundlagen der extrakorporalen Stosswellentherapie (ESWT) [physicaltechnical principles of extracorporeal shockwave therapy (ESWT)]. Orthopade. 2002;31:610-7.

8. Speed C. A systematic review of shockwave therapies in soft tissue conditions: focusing on the evidence. Br J Sports Med. 2014;48:1538-42.

9. Schmitz C, Császár NB, Milz S, Schieker M, Maffulli N, Rompe JD, Furia JP. Efficacy and safety of extracorporeal shock wave therapy for orthopedic conditions: a systematic review on studies listed in the PEDro database. $\mathrm{Br}$ Med Bull. 2015;116:115-38

10. Cacchio A, Giordano L, Colafarina O, Rompe JD, Tavernese E, loppolo F, et al. Extracorporeal shock-wave therapy compared with surgery for hypertrophic long-bone nonunions. J Bone Joint Surg Am. 2009;91:2589-97.

11. Furia JP, Juliano PJ, Wade AM, Schaden W, Mittermayr R. Shock wave therapy compared with intramedullary screw fixation for nonunion of proximal fifth metatarsal metaphyseal-diaphyseal fractures. J Bone Joint Surg Am. 2010;92:846-54. 
12. Notarnicola A, Moretti L, Tafuri S, Gigliotti S, Russo S, Musci L, et al. Extracorporeal shockwaves versus surgery in the treatment of pseudoarthrosis of the carpal scaphoid. Ultrasound Med Biol. 2010;36:1306-13.

13. Valchanou VD, Michailov P. High energy shock waves in the treatment of delayed and nonunion of fractures. Int Orthop. 1991;15:181-4.

14. Schleberger $R$, Senge T. Non-invasive treatment of long-bone pseudarthrosis by shock waves (ESWL(R)). Arch Orthop Trauma Surg. 1992;111:224-7.

15. Heinrichs W, Witzsch U, Bürger RA. Extrakorporale Stosswellentherapie (ESWT) von Pseudoarthrosen. Eine neue Indikation fur Regionalanasthesien. [extracorporeal shock-wave therapy (ESWT) for pseudoarthrosis. A new indication for regional anesthesia]. Anaesthesist. 1993:42:361-4.

16. Diesch R, Haupt G. Anwendung der hochenergetischen extrakorporalen Stosswellentherapie bei Pseudarthrosen [use of extracorporeal shock waves in the treatment of pseudoarthrosis]. Orthop Praxis. 1997;7:470-1.

17. Haupt G. Stosswellen in der Orthopädie [shock waves in orthopedics]. Urologe. 1997;36:233-8.

18. Haupt G. Use of extracorporeal shock waves in the treatment of pseudarthrosis, tendinopathy and other orthopedic diseases. J Urol. 1997;158:4-11.

19. Vogel J, Rompe JD, Hopf C, Heine J, Burger R. Die hochenergetische extrakorporale Stosswellentherapie (ESWT) in der Behandlung von Pseudarthrosen. [high-energy extracorporeal shock-wave therapy (ESWT) in the treatment of pseudarthrosis]. Z Orthop Ihre Grenzgeb. 1997;135:145-9.

20. Vogel J, Hopf C, Eysel P, Rompe JD. Application of extracorporeal shockwaves in the treatment of pseudarthrosis of the lower extremity. Preliminary results. Arch Orthop Trauma Surg. 1997;116:480-3.

21. Beutler S, Regel G, Pape HC, Machtens S, Weinberg AM, Kremeike I, et al. Extracorporeal shock wave therapy for delayed union of long bone fractures preliminary results of a prospective cohort study. Unfallchirurg. 1999;102:839-47.

22. Rompe JD, Rosendahl T, Schollner C, Theis C. High-energy extracorporeal shock wave treatment of nonunions. Clin Orthop Rel Res. 2001;387:102-11.

23. Schaden W, Fischer A, Sailler A. Extracorporeal shock wave therapy of nonunion or delayed osseous union. Clin Orthop Relat Res. 2001;387:90-4.

24. Wang CJ, Chen HS, Chen CE, Yang KD. Treatment of nonunions of long bone fractures with shock waves. Clin Orthop Rel Res. 2001:387:95-101.

25. Küfer R, Volkmer B, Nesslauer T, Schneider P, Hautmann RE, Gschwend JE. An innovative mobile lithotripter for extracorporeal shock wave lithotripsy and therapy. Urol Int. 2002;69:17-20.

26. Schatz KD, Nehrer S, Dorotka R, Kotz R. 3D-navigierte hochenergetische Stoßwellentherapie und Achskorrektur nach fehlgeschlagener Distraktionsbehandlung bei kongenitaler Tibiapseudarthrose [3D-navigated high energy shockwave therapy and axis correction after failed distraction treatment of congenital tibial pseudarthrosis]. Orthopade. 2002;31:663-6.

27. Biedermann R, Martin A, Handle G, Auckenthaler T, Bach C, Krismer M. Extracorporeal shock waves in the treatment of nonunions. J Trauma. 2003;54:936-42.

28. Chooi YS, Penafort R. Extra-corporeal shock-wave therapy in the treatment of non-unions. Med J Malaysia. 2004;59:674-7.

29. Schaden W, Fischer A, Sailler A. Extrakorporale Stoßwellentherapie (ESWT) aus der Sicht der Traumatologie [extracorporeal shock-wave therapy from viewpoint of traumatology]. J Mineralstoffwechsel. 2004;11:40-5.

30. Bara T, Synder M. Nine-year experience with the use of shock waves for treatment of bone union disturbances. Ortop Traumatol Rehabil. 2007;9:254-8.

31. Taki M, Iwata O, Shiono M, Kimura M, Takagishi K. Extracorporeal shock wave therapy for resistant stress fracture in athletes: a report of 5 cases. Am J Sports Med. 2007;35:1188-92.

32. Endres S, Weiskirch M, Hinz C, Hütter F, Wilke A. Extracorporeal shock-wave therapy in the treatment of pseudoarthrosis: a case report. Cases J. 2008;1:276.

33. Cacchio A, De Blasis E, Rosa F, De Blasis D, de Paulis F, Santilli V, et al. Response of bone turnover biochemical markers to extracorporeal shock wave therapy in the management of long-bone nonunions. Clin Chem. 2009;55:195-6.

34. Moretti B, Notarnicola A, Moretti L, Patella S, Tato I, Patella V. Bone healing induced by ESWT. Clin Cases Miner Bone Metab. 2009;6:155-8.

35. Wang CJ, Yang KD, Ko JY, Huang CC, Huang HY, Wang FS. The effects of shockwave on bone healing and systemic concentrations of nitric oxide (NO), TGF-(beta)1, VEGF and BMP-2 in long bone non-unions. Nitric Oxide. 2009:20:298-303.

36. Xu ZH, Jiang Q, Chen DY, Xiong J, Shi DQ, Yuan T, et al. Extracorporeal shock wave treatment in nonunions of long bone fractures. Int Orthop. 2009;33:789-93.
37. Elster EA, Stojadinovic A, Forsberg J, Shawen S, Andersen RC, Schaden W. Extracorporeal shock wave therapy for nonunion of the tibia. J Orthop Trauma. 2010;24:133-41.

38. Alvarez RG, Cincere B, Channappa C, Langerman R, Schulte R, Jaakkola J, et al. Extracorporeal shock wave treatment of non-or delayed union of proximal metatarsal fractures. Foot Ankle Int. 2011;32:746-54.

39. Stojadinovic A, Kyle Potter B, Eberhardt J, Shawen SB, Andersen RC, Forsberg JA, et al. Development of a prognostic naive bayesian classifier for successful treatment of nonunions. J Bone Joint Surg Am. 2011;93:187-94.

40. Vulpiani MC, Vetrano M, Conforti F, Minutolo L, Trischitta D, Furia JP, et al. Effects of extracorporeal shock wave therapy on fracture nonunions. Am J Orthop. 2012;41:E122-7.

41. Czarnowska-Cubała M, Gwoździewicz K, Studniarek M, Lasek J. Predictive role of scintigraphy (BS) in bone union induction using extracorporeal shock wave treatment (ESWT). J Orthop. 2013:10:70-3.

42. Alkhawashki HM. Shock wave therapy of fracture nonunion. Injury. 2015:46:2248-52.

43. Kuo SJ, Su IC, Wang CJ, Ko JY. Extracorporeal shockwave therapy (ESWT) in the treatment of atrophic non-unions of femoral shaft fractures. Int I Surg. 2015;24(Pt B):131-4

44. Haffner N, Antonic V, Smolen D, Slezak P, Schaden W, Mittermayr R, Stojadinovic A. Extracorporeal shockwave therapy (ESWT) ameliorates healing of tibial fracture non-union unresponsive to conventional therapy. Injury. 2016:47:1506-13.

45. Ikeda K, Tomita K, Takayama K. Application of extracorporeal shock wave on bone: preliminary report. J Trauma. 1999;47:946-50.

46. Ikeda K. Bone fracture and the healing mechanisms. Application of the extracorporeal shock wave on treatment of fracture. Clin Calcium. 2009:19:718-25.

47. Rompe JD, Eysel P, Hopf C, Vogel J, Küllmer K. Extrakorporale Stosswellenapplikation bei gestörter Knochenheilung. Eine kritische Bestandsaufnahme [Extracorporeal shockwave treatment of delayed bone healing. A critical assessment]. Unfallchirurg 1997;100:845-849.

48. Schoellner C, Rompe JD, Decking J, Heine J. High energy extracorporeal shockwave therapy (ESWT) in pseudarthrosis. Orthopade. 2002;31:658-62.

49. Bara T, Synder M, Studniarek M. The application of shock waves in the treatment of delayed bone union and pseudoarthrosis in long bones. Ortop Traumatol Rehabil. 2000;2:54-7.

50. Schaden W, Mittermayr R, Haffner N, Smolen D, Gerdesmeyer L, Wang CJ. Extracorporeal shockwave therapy (ESWT)-first choice treatment of fracture non-unions? Int J Surg. 2015;24:179-83.

51. Silk ZM, Alhuwaila RS, Calder JD. Low-energy extracorporeal shock wave therapy to treat lesser metatarsal fracture nonunion: case report. Foot Ankle nt. 2012:33:1128-32

52. Gollwitzer H, Gloeck T, Roessner M, Langer R, Horn C, Gerdesmeyer L, et al. Radial extracorporeal shock wave therapy (rESWT) induces new bone formation in vivo: results of an animal study in rabbits. Ultrasound Med Biol. 2013;39:126-33.

53. Diaz-Rodriguez L, Garcia-Marinez O, Arroyo-Morales M, Ramos-Torrecillas J, De Luna-Bertos E, Ruiz C. Effect of radial extracorporeal shock wave therapy on proliferation, cell viability and phagocytosis of human osteoblasts (MG63). Adv Sci Lett. 2012:17:325-9.

54. Chitnis PV, Cleveland RO. Acoustic and cavitation fields of shock wave therapy devices. In: therapeutic ultrasound: 5 th international symposium on therapeutic ultrasound. AIP Conf Proc. 2006;829:440-4.

55. Császár NB, Angstman NB, Milz S, Sprecher CM, Kobel P, Farhat M, Furia JP, Schmitz C. Radial shock wave devices generate cavitation. PLoS One. 2015;10:e0140541.

56. Angstman NB, Kiessling MC, Frank HG, Schmitz C. High interindividual variability in dose-dependent reduction in speed of movement after exposing C. Elegans to shock waves. Front Behav Neurosci. 2015;9:12.

57. Ogden JA, Tóth-Kischkat A, Schultheiss R. Principles of shock wave therapy. Clin Orthop Relat Res. 2001:387:8-17.

58. Phieffer LS, Goulet JA. Delayed unions of the tibia. J Bone Joint Surg Am. 2006:88(1):206-16.

59. Frölke JP, Patka P. Definition and classification of fracture non-unions. Injury. 2007:38(Suppl 2):S19-22

60. Flierl MA, Smith WR, Mauffrey C, Irgit K, Williams AE, Ross E, Peacher G, Hak $D J$, Stahel PF. Outcomes and complication rates of different bone grafting modalities in long bone fracture nonunions: a retrospective cohort study in 182 patients. J Orthop Surg Res. 2013:8:33.

61. https://www.fda.gov/ohrms/dockets/98fr/98d0238.pdf. Accessed 3 Nov 2017. 
62. Tischer T, Milz S, Weiler C, Pautke C, Hausdorf J, Schmitz C, et al. Dose-dependent new bone formation by extracorporeal shock wave application on the intact femur of rabbits. Eur Surg Res. 2008:41:44-53.

63. Hofmann A, Ritz U, Hessmann MH, Alini M, Rommens PM. Extracorporeal shock wave-mediated changes in proliferation, differentiation, and gene expression of human osteoblasts. J Trauma. 2008;65:1402-10.

Submit your next manuscript to BioMed Central and we will help you at every step:

- We accept pre-submission inquiries

- Our selector tool helps you to find the most relevant journal

- We provide round the clock customer support

- Convenient online submission

- Thorough peer review

- Inclusion in PubMed and all major indexing services

- Maximum visibility for your research

Submit your manuscript at www.biomedcentral.com/submit 